\title{
"Charisma macht müde Demokratien munter" - Zum diskursiven Zusammenhang von Charisma und Demokratie
}

\author{
Pamela Steen (Leipzig)
}

\begin{abstract}
"I am charismatic!". If politician described themselves like this, we would probably define them as a peacock and condemn such a vain display. "Barack Obama is charismatic" or "Charisma perks up tired democracies", however, are propositions we can read in newspapers every day. Although they are assumptions about fundamental agencies, they are scarcely questioned. But these ascriptions or assertions must be believed or not, they are elements in a complex constructed belief system which is called politics. This paper employs the method of discourse linguistics to demonstrate how the relation of charisma and democracy can be construed in media discourse. Three main positions can be extracted: Charisma is inevitable for genuine democratic processes like functions and elections. Charisma is useful to overcome post-democratic challenges, and thirdly, charisma is incompatible with democracy, because charisma is an illusion or a pre-democratic phenomenon. In summary, there are different concepts of charisma and democracy, which collude, depending on the argumentation.
\end{abstract}

\section{$1 \quad$ Charisma als sprachlich-mediales Konstrukt}

"Barack Obama ist charismatisch" oder "Charisma macht müde Demokratien munter" sind Diskursaussagen, die kaum angezweifelt werden, obwohl diese Fremdzuschreibungen konstruierte Glaubenssätze sind. Heißt es einige Jahre später in den Medien "Barack Obama hat sein Charisma verloren", so glauben die Rezipienten vermutlich auch diese These - vorausgesetzt, es können genügend stichhaltige Argumente dafür angeführt werden.

Soziologie und Politikwissenschaft fragen danach, welche gesellschaftlichen Umstände dazu beitragen, dass die Sehnsucht nach Charismatikern wächst und welche Eigenschaften oder Fähigkeiten es sind, die an solchen Politikern als charismatisch beurteilt werden - die andere Menschen folglich zu Charisma-Gläubigen machen. Nach Max Weber (1922: 246), an dessen Herrschaftstypologie sich die moderne Charisma-Forschung weitgehend orientiert, ist Charisma "eine schlechthin an dem Objekt oder der Person, die es nun einmal von Natur besitzt, haftende, durch nichts zu gewinnende Gabe". Dadurch gerät die charismatische Person zum Mittelpunkt der Forschung (vgl. Marschall 2011; Steyrer 2011). ${ }^{1}$ Damit es zu einer intersub-

\footnotetext{
1 Die mittlerweile unüberschaubar gewordene sozialwissenschaftliche Charisma-Forschung ist um einiges komplexer und differenzierter als dies hier in der Kürze wiedergegeben werden kann. So befragt sie etwa auch Linguistik Online 73, 4/15 - http://dx.doi.org/10.13092/lo.73.2198
}

CC by 3.0 
jektiven Einschätzung und Bewertung eines Politikers als charismatisch kommt, bedarf es in der Moderne und Postmoderne zunehmend einer erfolgreichen medialen Performanz dieses Politikers, kurz: einer Medialisierung von Macht (vgl. Tänzler 2000). Daher rücken ebenso vermehrt rhetorische Fähigkeiten und Inszenierungsmechanismen von Politikern in den Blick (vgl. Lenze 2002), die Politik emotionalisieren und personalisieren. Dieses telegene Charisma wird als eine Art degeneriertes Charisma und als "Ware auf dem Medienmarkt" (Fasel 2001: 64) verstanden. Die Einschätzung, ob jemand charismatisch sei, ist jedoch trotz aller medialer Inszenierungstechniken genauso wenig oktroyierbar, wie sie objektiv ist.

Aus dieser sozialwissenschaftlichen Auffassung von Charisma ergibt sich eine Forschungsfrage für die Diskurslinguistik (Spitzmüller/Warnke 2011; Busse/Teubert 1994) und ein grundlegendes Desiderat. Die bisherige Forschung zur charismatischen Persuasion fokussiert vor allem die von dem Politiker genutzten rhetorischen wie medialen Mechanismen. ${ }^{2}$ Die Verbindung zwischen einer erfolgreichen Person des öffentlichen Lebens und einer ihr zujubelnden Masse wird als "charismatische Beziehung" bezeichnet: "Worin gründet die charismatische Macht von Madonna?" fragt etwa Stephen Turner (2007: 93). Eine solche Forschungsfrage beinhaltet bereits die Zuschreibung einer charismatischen Beziehung, wobei den Akteuren, auf die Bezug genommen wird, Madonna und ihren Fans, der Begriff Charisma gar nicht geläufig sein muss. Ausgeblendet wurde bislang, welche Rolle mediale Texte spielen, in denen solche Beziehungen sprachlich als empirische Entität (erst) konstruiert werden.

Wenn etwa Tageszeitungen einen aufstrebenden Politiker als "charismatisch" bezeichnen, so spiegelt diese sprachliche Zuschreibung nicht wider, dass dessen mediale Inszenierung erfolgreich war. Vielmehr findet in Zeitungsartikeln, Talkshows und Internetforen (sowie übrigens auch in soziologischen Texten) die eigentliche - höchst uneinheitliche - Konstruktion von Charisma als gesellschaftlichem Wissensaspekt erst statt. Erst im Diskurs wird das Wissen "immer wieder neu durch Akteurshandlungen respektive argumentative Thematisierung produziert" (Spitzmüller/Warnke 2011: 58). Die Performanz des Politikers ist zwar eine mögliche Voraussetzung für seine Wahrnehmung als charismatisch, jedoch nicht Teil des Diskurses über Charisma. Charisma ist aus dieser Perspektive nicht als ein ontologisch verankerter Wissensaspekt anzusehen, der "seine Legitimierung in den res extensa (der Welt der wahrnehmenden Umwelt) selbst findet" (Spitzmüller/Warnke 2011: 58). Max Webers (1922: 246) Formulierung, dass der Charismaträger seine Gaben "von Natur besitzt" könnte zu einer anderen Auffassung verleiten. Dass es bei der medialen Zuschreibung von Charisma aber um etwas anderes geht als darum, die Realität sprachlich abzubilden, zeigen allein systematische Inkohärenzen in der Zu- und Abschreibung: Beispielsweise galt der ehemalige Bundesverteidigungsminister Karl Theodor zu Guttenberg nicht schon immer als charismatisch; er galt es aufgrund einer, ebenfalls durch die Medien beeinflussten, größeren Popularität, jedoch nicht für alle Medienakteure unisono (es gab auch Gegenstimmen); und man schrieb ihm sein Cha-

\footnotetext{
historische gesellschaftliche Wandlungsprozesse auf ihren Zusammenhang mit Charisma (vgl. Breuer 1993), die Theorie Webers wird präzisiert, ausgeweitet, verändert, und es werden Möglichkeiten der Charisma-Genese untersucht, z. B. als Resultat einer Selbststigmatisierung (vgl. Lipp 1993).

2 Für die Diskursanalyse ist hier besonders der massenmediale Diskurs von Interesse. Diskurs wird hier textualistisch als transtextuelle Sprachstruktur verstanden (vgl. Spitzmüller/Warnke 2011).
}

ISSN 1615-3014 
risma wieder ab, nachdem in den Medien Plagiats-Vorwürfe erhoben wurden, die gleichsam zu einem Charismavernichter wurden.

Der Glaube an Charisma wird wie bei allen sozialen Konstruktionen der Wirklichkeit kommunikativ etabliert (vgl. Berger/Luckmann 2009). Jentges (2010: 77) nennt dies "Charismatisierungsprozesse", die Gemeinschaft der Zuschreibenden bezeichnet er als "Interpretationsgemeinschaft". Mit einer diskurslinguistischen Herangehensweise, die die Praxis der Interpretationsgemeinschaft systematisch untersucht, erhält die Charisma-Forschung eine zusätzliche kritische Perspektive hinsichtlich einer pragmatisch verankerten Grundlage von Charisma. Es ist z. B. danach zu fragen, wem eine $\mathrm{Zu}$ - oder Abschreibung von Charisma nützt, etwa bei anstehenden Wahlen, und welche Argumente dafür ins Feld geführt werden.

Der Zusammenhang zwischen Charismatisierungen und Faktoren des politischen Systems kann dabei in Diskursaussagen mehr oder weniger explizit formuliert sein: Die Headline "Charisma macht müde Demokratien munter" (Berliner Republik online, 01.03.2014) zielt direkt auf die Verbindung von Charisma und System ab. Dabei wird präsupponiert, dass Charisma eine wie auch immer beschaffene ontologische Entität sei. Charisma besetzt dabei die Position des Agens; aufgrund einer semantischen Verschiebung steht der Ausdruck metonymisch für den Charismaträger. Die Grundaussage des Satzes lautet demnach: Charismatische Politiker (deren Charisma als ontologisch existent betrachtet wird) haben die Fähigkeit, müde gewordene Demokratien zu revitalisieren. Dabei wird impliziert, dass Demokratien müde geworden sein können oder zumindest dazu neigen können, es zu werden. Im Artikel selbst distanziert sich der Autor von dieser Auffassung und führt mehrere Gründe dafür an, warum Charismatiker kein Allheilmittel "in Zeiten der Mediendemokratie" (ebd.) sein können. Damit wird Charisma als Gegenstand zum Thema und als politisches Phänomen im Hinblick einer speziellen demokratischen Ausprägung problematisiert: Quellen dieser Art erscheinen daher als thematisierende Texte.

Häufiger lassen sich Medien-Texte finden, in denen die Ausdrücke Charisma, charismatisch oder deren Synonyme als nicht weiter zu hinterfragende oder zu definierende Referenzausdrücke verwendet werden (Okkurrenz-Texte). Eher selten hingegen sind metasprachlichreflexive Texte, in denen Diskursakteure die sprachlich-mediale Konstruktion von Charisma reflektieren und damit ihre eigene Rolle bei der Zuschreibung von Charisma (metasprachlichreflexive Texte). Wo eine solche Zuschreibungspraxis nicht offengelegt wird - mithin in thematisierenden Texten und Okkurenztexten - sind Charismatisierungen oft Ausdruck einer (durchaus zum politischen Alltag gehörenden) Sakralisierung und Mystifizierung.

\section{Sakralisierung und Mystifizierung des politischen Diskurses}

Im Duden wird der Ausdruck Charisma als polysem aufgeführt. Seine beiden Bedeutungen sind 1. 'die durch den Geist Gottes bewirkten Gaben und Befähigungen des Christen in der Gemeinde (Theol.)' und 2. die 'besondere Ausstrahlungskraft eines Menschen'. Dagegen machen Diskursaussagen wie "Barack Obama - der Messias von Greenwood" (Die Welt online, 24.01.2008) oder "Barack Obama - Politiker, kein Messias" (Stuttgarter Zeitung, 21.01.2013) in der Ausdrucksverwendung durch ihre systematisch eingesetzten referenzidentischen Metaphern aus dem religiösen Bereich eine ambige Bedeutungsbeziehung deutlich. Virulente Ausdrücke wie Messias, Heilsbringer, Lichtgestalt, Hoffnungsträger, Glaube, die in unterschied- 
licher Funktion bei der sprachlichen Charismatisierung von Politikern verwendet werden, indizieren einen insgesamt religiös aufgeladenen Diskurs. Auch wenn Obama nicht im dezidiert christlichen Sinne als Wiedergeburt Jesu verstanden wird, so werden oder wurden doch mit seinen "Visionen" Hoffnungen auf eine andere, neue amerikanische Politik verknüpft und diese wiederum im Diskurs in unterschiedlicher Weise mit Argumentationen pro oder contra Obama verbunden. Hoffnungen aber referieren auf etwas Zukünftiges, an dessen Eintreten man glauben muss, enttäuschte Hoffnungen auf die Gegenwart, die den in der Vergangenheit gesetzten Glauben nicht bestätigt hat.

Charismatisierungen zeigen, dass Politik kein durch und durch rationales System ist. Politik ist ein Glaubenssystem, auch wenn sich die politischen Wirkkräfte im 21. Jahrhundert pluralisiert haben; Yves Bizeul (2009: 27) sieht daher in Barack Obamas "Yes we can"-Bewegung einen Beweis für die "Persistenz des politischen Glaubens nach dem Ende der großen politischen Ideologien und Utopien". Für Bizeul sind die Grenzen zwischen dem religiösen und dem politischen Glauben fließend. Als Instanz, die Wissen mit einem allgemeinen Geltungsund Wahrheitsanspruch distribuiert, sind die Religionen zumindest in der westlichen Welt seit der Moderne kaum noch relevant. Die Religion "wirkt an der Wissensbegründung nicht mehr mit" (Bizeul 2009: 35; vgl. Luhmann 2000: 315). Religiöse Metaphern, Inhalte und Topoi in politischen Diskursen indes sind Hinweise darauf, dass eine rationale Sakralisierung des Subjekts an der Wissensbegründung beteiligt ist. ${ }^{3}$ Auch die sozialwissenschaftliche Forschung sieht den Charisma-Begriff als einen "nicht sozialisierbaren Rest des menschlichen Handelns" (Constans Seyfarth, zit. nach Gebhardt/Zingerle/Ebertz 1993: V), der auf Tatbestände verweist,

die sich jeder vordergründigen sozialstrukturellen Erklärung entziehen, vielmehr in jenem weiten und diffusen Feld menschlicher Emotionen, Glaubensüberzeugungen und Zukunftshoffnungen angesiedelt sind $[\ldots]$.

(Gebhardt/Zingerle/Ebertz 1993: V)

In den Medien muss die Konstruktion des Charisma-Begriffs als kaum hinterfragbarer Glaubensaspekt jedoch nicht immer explizit über religiöse Metaphern erfolgen. PseudoErklärungen und -Definitionen verdunkeln den Begriff eher, als dass sie ihn beleuchten. So wird Charisma häufig mit einer Aneinanderreihung paraphrasierender Ausdrücke erklärt. Häufig kommt dann eine Eigenschaft x hinzu, die die Attribute als geheimnisvoller Kitt verbindet und damit aus der Summe der Teile eine neue Qualität macht:

Es ist die Hoffnung auf eine Person, die Emotionen auslöst und Empathie weckt. Es verlangt uns Deutsche nach einer markanten Persönlichkeit, die Würde, Erfahrung, Aura, Unabhängigkeit, Ausdruckskraft und Begeisterungsfähigkeit in sich vereint.

(Steg 2010)

Der ehemalige Regierungssprecher Thomas Steg erklärt Charisma zunächst mit der Wirkung einer charismatischen Person, um dann Eigenschaften aufzuzählen, die der Politiker haben muss, um diese Wirkung zu erzielen. Es folgt eine Reihe von Hochwertwörtern, die vage

\footnotetext{
3 Vgl. den Begriff des Heiligen bzw. des Sakralen bei Otto (1917) bzw. Rappaport (1979/2013: 207): "Es ist interessant, dass sakrale Annahmen und numinose Erfahrungen gegenteilig sind. Letztgültige sakrale Annahmen sind diskursiv, ihre Referenzbezüge aber sind nicht-materiell".
} 
bleiben, schwer greifbar und individuell sind (markante Persönlichkeit, Würde). Ausdruckskraft und Erfahrung referieren wiederum auf Eigenschaften, die von einem Politiker normalerweise erwartbar sind. Mit Unabhängigkeit wird eine Position und Agency des Politikers konstruiert, die im Hinblick auf demokratische und medialisierte Machtstrukturen kaum einlösbar ist, sondern als idealistisch zu werten ist. Stegs Aneinanderreihung zeigt, dass Charisma kaum erklärt, nur durch eine Vielzahl an Hochwertwörtern umschrieben wird. Vor allem aber die Verwendung des Ausdrucks Aura, dessen Bedeutung im Duden ebenfalls u. a. mit 'Ausstrahlung' angegeben wird, macht aus der Umschreibung eine versteckte Tautologie, die gerade dadurch ihre mystifizierende Kraft erhält. Mit der Generalisierung uns Deutsche unterlegt der Akteur zudem dem gesamten Volk diese Definition von Charisma und konstruiert damit eine starke Lobby für seine Position.

Auch Altbundespräsident Roman Herzog erklärt Charisma in einem Zeitungsinterview von seiner Wirkung her:

Die Welt, in der wir leben, wird immer unverständlicher, die Veränderungen kommen immer schneller. Die Gründe dafür kann man alle erklären. Sehr oft aber können wir nicht auf eine tiefgründige Analyse warten, sondern müssen vorher handeln. Dadurch entsteht immer mehr Unsicherheit. Der im Augenblick handelnde Politiker braucht also viel Vertrauen. Dazu gehören Ehrlichkeit und Erfolg. Dem charismatischen Politiker wird von den Bürgern automatisch größeres Vertrauen entgegengebracht. Deshalb sind solche Politiker jetzt so wichtig.

(Herzog 2009)

Herzog konstruiert mit dem Argument der Komplexität eine Notwendigkeit für Politiker, die im Augenblick handeln und ehrlich sind. In Charisma thematisierenden Texten ist der Komplexitäts-Topos eine häufige Denkfigur, ein "Denkmuster" (vgl. Bornscheuer 1976; Wengeler 2013: 198-201), das je nach Argumentation pro und contra Charisma eingesetzt wird. In Herzogs Argumentation macht die Komplexität der Gesellschaft charismatische Politiker notwendig, da diesen automatisch Vertrauen entgegengebracht wird, so dass sie im Augenblick handeln können. Der geheimnisvolle Kitt, der aus einem normalen einen charismatischen Politiker macht, steckt hier im Adverb automatisch. Herzog sagt nicht, wie es dazu kommt, dass dem charismatischen Politiker Vertrauen entgegengebracht wird, warum er also charismatisch ist, sondern er argumentiert für die Wichtigkeit von Charismatikern mit ihrer Wirkung, ohne zu erklären, wie diese erzielt wird. Im Gegenzug wird aber nicht jeder ehrliche, erfolgreiche und im Augenblick handelnde Politiker in den Medien automatisch als charismatisch bezeichnet. Notwendige Bedingungen werden genannt, hinreichende bleiben im Dunkeln.

In einem Geleitwort zu Lenze (2002: V) spricht Jo Reichertz von einer "Nicht-Vertextbarkeit" von Charisma, insofern als Charisma aufgrund von "nicht textbasierten Zeichen" zugeschrieben wird. Die Beispiele bestätigen, dass Charisma schwerlich vertextbar ist, und sie zeigen auch, dass diese Nicht-Vertextbarkeit nicht nur auf der Unfähigkeit der Akteure beruht, sondern System hat, denn

Charisma (...) bedarf (...) eines geheimnisvollen Kerns, der gewissermaßen als charismatischer Profanisierungs- und Kopierschutz dient, demnach die Demokratisierung charismatischer Befähigung für jedermann verhindert und gleichzeitig ein Begehren produziert, das sich auf das Geheimnis richtet, ohne dieses jemals ganz lüften zu können. 
Wenn pro Charisma argumentiert wird, dann müsste die "Demokratisierung charismatischer Befähigung" ein hehres Ziel sein; tatsächlich aber wird Charisma in den Medien als knappes Gut konstruiert, obwohl allenthalben davon die Rede ist. In der Art und Weise, wie von Charisma die Rede ist, wie also die Vertextung stattfindet, wird deutlich, dass ein nicht greifbarer Heilsbringer und Welterklärer konstruiert wird, der den Wunsch nach Wiederverzauberung erfüllt. Vertextet wird durch explizite Sakralisierung (ohne Spiritualität) und implizite Mystifizierung (ohne Sakralisierung). Die Sehnsucht nach dem Numinosen, die durch kirchliche Institutionen nicht mehr adäquat gestillt werden kann, diffundiert nicht nur in die private Religion (vgl. Knoblauch 2009), sondern auch in die Politik. Dort haben irdische Heilsbringer konkrete Aufgaben zu lösen, weshalb Dirk Tänzler (2007: 128) in der Moderne eine Entwicklung

von der Verkörperung einer transzendenten Kraft in der Person zur Charismatisierung der Person selbst als der letzten, innerweltlichen Quelle des Heiligen

(Tänzler 2007: 128)

sieht. Auf den Charismatiker wird eine immanente Transzendenz projiziert, die im Sinne von Alfred Schütz und Thomas Luckmann (2003: 597-609) zwischen einer "mittleren" und einer "großen" Transzendenz liegt. ${ }^{4}$

Es kommt nun darauf an, die Strukturen der Diskurse, die durch die Interpretationsgemeinschaft hervorgebracht werden, daraufhin zu untersuchen,

wie der Geltungsanspruch für eine solche transzendente Macht kommunikativ erzeugt und wie in Bezug auf diesen "subjektiv gemeinten Sinn" soziale Beziehungen strukturiert werden.

(Tänzler 2007: 123)

Die von Tänzler angesprochenen "sozialen Beziehungen", die durch Charismatisierungen strukturiert werden, werden deutlicher als in Texten noch in direkten verbalen Interaktionen, wie z. B. TV-Talkshows. ${ }^{5}$

\section{Zum Zusammenhang der Begriffe Charisma und Demokratie im Diskurs}

Wenn Demokratie als Regierungssystem gedacht ist, in dem das Volk über frei gewählte Vertreter indirekt an der Machtausübung im Staat teilhat, sind Charisma und Demokratie dann Phänomene, die miteinander vereinbar sind? Diese Frage stellen sich Soziologen in Bezug auf

\footnotetext{
${ }^{4}$ Immanent ist diese Transzendenz, weil sie nicht aufgrund einer Dichotomie zwischen dem Profanen und dem Heiligen entsteht, sondern im irdischen Wesen selbst angesiedelt ist. Schütz/Luckmann (2003: 589-633) unterscheiden drei Arten von Transzendenz: Im Zuge kleiner Transzendenzen setzt sich das Ich selbstreflexiv mit sich und seiner Umwelt auseinander; mittlere Transzendenzen entstehen, wenn das Ich mit einem Du konfrontiert wird, wenn es sich in den Anderen hineinversetzt; große Transzendenzen sind andere Wirklichkeiten wie Ekstase oder Traum.

5 So lassen sich z. B. in der Talkshow "Maischberger" mit dem Titel "Der Schummelbaron - Frechheit siegt?" vom 22.02.2011 die Diskursakteure klar den Fraktionen "pro Guttenberg" und "contra Guttenberg" zuteilen, wenn es um die Frage geht, ob der Verteidigungsminister aufgrund der Plagiatsaffäre zurücktreten soll. Dabei ist die gesamte Diskussion von der vorherigen Charismatisierung Guttenbergs beeinflusst. Seine Fallhöhe ist so hoch, dass die Pro-Akteure Argumente dafür vorbringen können, einen solchen Ausnahmepolitiker halten zu müssen, während die Contra-Akteure gerade aufgrund der Verfehlungen die Charismatisierung als nachträgliche Fehleinschätzung desillusionieren - ohne dass der Ausdruck Charisma überhaupt fällt.
} 
Webers Charisma-Konstruktion als Herrschaftstypus und deuten dessen Schriften ganz unterschiedlich aus (vgl. Tänzler 2007; Breuer 1991). Diese soziologische Grundsatz-Diskussion kann hier nicht im Einzelnen nachgezeichnet werden, zumal der Charisma-Begriff, wie erläutert, auch in der Soziologie ganz unterschiedlich aufgefasst wird. Für eine mediendiskurslinguistische Perspektive ist die Frage relevant, ob Diskursakteure die von ihnen konstruierten Begriffe Charisma und Demokratie für miteinander vereinbar halten. Je nachdem, wie die Antwort ausfällt, trägt dies zu einem besseren Verständnis ihrer diskurssemantischen Bedeutungen bei. Wird Charisma beispielsweise für unverträglich mit demokratischen Prozessen gehalten, so ist wahrscheinlich, dass das Konzept von Charisma kaum die auf bloße Äußerlichkeiten reduzierte 'Ausstrahlung' ist, sondern Implikationen von 'Macht' oder 'Herrschaft' besitzt, die mit der Auffassung von einer Volkssouveränität kollidieren. Ob der Zusammenhang der Begriffe explizit problematisiert wird oder dieser vom Leser inferiert werden muss, gibt Aufschluss darüber, welcher Grad an Reflexivität vorhanden ist bzw. welches generelle Problembewusstsein hinsichtlich der Charismatisierungsprozesse rekonstruiert werden kann. Anschließend an die Überlegungen zur Sakralisierung und Mystifizierung personaler Eigenschaften von Politikern scheinen Charismatisierungen jedoch gerade vor dem Hintergrund eines demokratischen Systems eine besondere Relevanz zu besitzen. Das konstruierte Charisma repräsentiert eine transzendente und gleichzeitig immanente Macht, die eine politische Repräsentations- oder Machtbeziehung, die möglicherweise erst durch Wahlen gefestigt werden muss, legitimiert (vgl. Tänzler 2007: 123).

Im Folgenden bilden thematisierende und Okkurrenz-Texte die Analysegrundlage für eine exemplarische diskurslinguistische Untersuchung des Zusammenhangs von Charisma und Demokratie als Diskursphänomene. Den Schwerpunkt bildet eine propositionsorientierte Analyse (vgl. Spitzmüller/Warnke 2011: 145-157) mit einem Fokus auf der Rekonstruktion von Argumentations-Topoi (vgl. Kienpointner 1993) und Redehintergründen (vgl. Zifonun 1997: 1882-1887). Dabei wird rekonstruiert, welche grundsätzlichen Bewertungen von Charisma im Hinblick auf seinen ontologischen Status und seine Bedeutung für demokratische Prozesse vorgenommen werden.

Die Analysen basieren auf einem kleinen Korpus, das für diese Untersuchung zusammengestellt wurde und Teil eines größeren Korpus zur Untersuchung von Charisma-Konstruktionen und -Konzepten im Diskurs ist, das (in anderem Zusammenhang) auch hinsichtlich quantitativer Aspekte ausgewertet wird. Für die Rekonstruktion des Zusammenhangs von Charisma und Demokratie wurden zunächst 96 Online-Artikel aus einem Zeitraum von 2008-2014 ausgewertet. ${ }^{6}$ Hierfür wurden auf den frei zugänglichen Online-Portalen großer deutscher überregionaler Zeitungen (taz, Zeit Online, Bild, Frankfurter Rundschau online, Handelsblatt online, Tagesspiegel, Süddeutsche, Spiegel online) die Suchbegriff-Kombinationen "Charisma + Demokratie", "Charisma + Wahlen", "Charisma + Politik" eingegeben. Damit ist klar, dass

\footnotetext{
${ }^{6}$ Dieser Zeitraum ist aus verschiedenen Gründen für eine Untersuchung von Charisma relevant: Obama wurde als erster schwarzer Politiker US-Präsident und auch von deutschen Medien zum politischen Messias stilisiert; in Deutschland wurde Guttenberg zum Heilsbringer der Union hochgeschrieben und dann aufgrund seines Plagiats de-charismatisiert mit direkten Versuchen der Re-Charismatisierung; im Zuge der Wahl zum Bundespräsidenten, den Bundestagswahlen und im Zusammenhang mit der Wirtschaftskrise fiel der Ausdruck Charisma ebenfalls signifikant oft.
} 
nicht alle in diesem Zeitraum für die Untersuchung möglicherweise relevanten Artikel erfasst wurden, sondern die berücksichtigten Texte eine systematische Stichprobe repräsentieren.

Die untersuchten Texte lassen sich nach drei Kategorien des Charisma-DemokratieZusammenhangs einteilen:

- Charisma ist notwendig für die Demokratie.

- Charisma ist nützlich für die Demokratie.

- Charisma ist unvereinbar mit Demokratie.

1) Charisma ist eine für demokratische Prozesse notwendige Eigenschaft von Politikern.

$\mathrm{Zu}$ dieser Haltung gibt es eine affirmierende und eine postulierende Variante. 1. Die affirmierende Haltung gründet sich auf der expliziten oder impliziten Feststellung, dass Charisma in der deutschen Politik vorhanden ist. 2. Bei der postulierenden Variante wird zunächst ein Mangel an Charisma in der deutschen Politik (oder bei einem bestimmten Politiker) konstruiert, sodass die Abwesenheit von Charisma und die damit einhergehenden Probleme negativ bewertet werden. Beiden Varianten ist ein "normativer Redehintergrund" (Zifonun 1997: 1884) eigen, da es sich hierbei meist um eine generalisierende Sprechweise handelt. Die folgenden vier Beispiele demonstrieren diese im Zusammenhang mit Demokratie eher unkritische Haltung, die charismatische Politiker grundsätzlich für wählbarer und wirksamer hält als nicht-charismatische Politiker.

(1) Affirmierend und postulierend: Am 08.05.2012 titelt das Handelsblatt online "Lindner und Kubicki verbindet nur das Charisma". Nach der für die FDP erfolgreichen Landtagswahl in Schleswig-Holstein wird ihrem Kandidaten Wolfgang Kubicki Charisma bescheinigt. Auch Christian Lindner, der Spitzenkandidat der Partei in Düsseldorf hat demnach Charisma. An ihrem Charisma muss sich der Parteichef Philipp Rösler messen lassen:

Eine Stärke allerdings verbindet Kubicki und Lindner. Beide sind Wahlkämpfer an der Spitze ihrer Landesverbände, die das Interesse der Bürger auf sich ziehen. Politiker mit einem Charisma, das Parteichef Philipp Rösler nicht entwickelt hat. Eine Eigenschaft, die für die Spitzenkandidatur bei der Bundestagswahl aber eine wichtige Rolle spielen wird.

(Sigmund 2012)

Das Charisma von Kubicki und Lindner wird affirmierend dem fehlenden Charisma von Rösler gegenübergestellt, dessen Charisma-Notwendigkeit postuliert wird. Da für Rösler konstatiert wird, er habe Charisma "nicht entwickelt", wird zudem impliziert, dass Charisma keine natürliche Gabe, sondern herstellbar ist. Damit liegt das vorweggenommene Scheitern Röslers bei der Bundestagswahl in dessen Verantwortung. Mit der Nominalphrase "Interesse der Bürger" wird zudem eine Folge von Charisma und nicht sein Wesen oder dessen Herstellbarkeit erklärt. Mystifizierend dargestellt wird daher der Zusammenhang von Charisma und einem Wahlsieg, wobei Charisma hierfür eine "bewirkende Ursache" (vgl. Kienpointner 1993: 335) ist. Nach Kienpointner (1993: 335) handelt es sich dabei um "eine Kombination von Handlungen eines Agens und dadurch ausgelösten kausalen Ketten", nur dass in dem angeführten Beispiel die notwendige Handlung des Agens nicht benannt und nur die kausale Folge Interesse der Bürger angegeben wird. Charisma als "Eigenschaft" spielt also eine "wichtige Rolle" bei grundlegenden demokratischen Prozessen wie Wahlen; Charisma wird als notwendig angesehen und positiv bewertet. Es wird gleichsam zum Totschlagargument gegen Rösler, 
gegen den keine anderen Verfehlungen, als eben kein Charisma "entwickelt" zu haben, vorgebracht werden.

(2) Affirmierend: Am 03.06.2010 wurde Christian Wulff als Bundespräsidentschaftskandidat der Regierungskoalition aus CDU/CSU und FDP vorgestellt. Aus diesem Grund gibt es an diesem Tag viele Kommentare, die die Chancen von Wulff bei der Wahl zum Thema machen. In der Zeit bewertet die Kommunikations- und Politikwissenschaftlerin Andrea Römmele den Kandidaten Wulff und ist dabei eine der wenigen Stimmen, die dem "Double für Herrn Kaiser" (taz, 25.06.2010) Charisma bescheinigen.

Christian Wulff hat die persönliche Ausstrahlung und das Charisma, das ein Bundespräsident braucht, um sich Gehör zu verschaffen.

(Römmele 2010)

Mit dieser Aussage konstruiert die Akteurin Charisma vor einem normativen Redehintergrund, da der Besitz von Charisma ("Wulff hat") im Hinblick auf das demokratische Amt des Bundespräsidenten als notwendig und daher als "materielle Ursache" (Kienpointner 1993: 335) für eine erfolgreiche Amtsausübung erachtet wird. Deutlich wird dies durch die Verwendung des Verbs brauchen. Nur ein charismatischer Politiker kann sich Gehör verschaffen, wird also überhaupt erst vom Volk und von der Politik wahrgenommen. Diese Aussage impliziert gleichwohl, dass das Amt allein nicht ausreicht, um als Bundespräsident wirksam zu sein. Insgesamt handelt es sich, bezogen auf die zukünftige Präsidentenwahl, um die Vorhersage einer konkreten kausalen Wirkung, denn ob Wulff sich tatsächlich wird Gehör verschaffen können, kann die Akteurin nicht wissen. Die Bedeutung von Charisma kann hier (anders als im Duden angegeben) nicht 'Ausstrahlung' sein, da die Konjunktion und beide Ausdrücke als gleichwertige zu besitzende Eigenschaften nebeneinander stellt. Was die Akteurin unter Charisma versteht, erklärt sie weiter oben mit einem Weber-Zitat:

Dafür muss er einen Stil entwickeln, der das beinhaltet, was Max Weber in seinen Ausführungen als Charisma bezeichnet hat. Denn in den Worten Webers qualifiziert sich ein Politiker in erster Linie durch Leidenschaft, Verantwortungsgefühl und Augenmaß.

(Römmele 2010)

Max Weber wird im medialen Diskurs häufig im Rahmen des Autoritäts-Schemas (vgl. Kienpointner 1992: 393-402) herangezogen, um den schwierigen Sachverhalt Charisma zu erklären. Neben Sakralisierung und Mystifizierung ist dies der Modus der Rationalisierung: Charisma wird vermeintlich wissenschaftlich fundiert definiert. In diesem Beispiel wird anachronistisch und ausschnitthaft über das Zitat der Eindruck erweckt, Webers CharismaBegriff ließe sich eins zu eins auf die heutige Zeit als ausschließlich positiv besetzter Begriff übertragen. Tatsächlich stellt Weber in seinem Vortrag "Politik als Beruf" den charismatischen Politiker einerseits dogmatisch einem faden Berufspolitiker gegenüber, andererseits ist der charismatische Führer nur mit einer seelenlosen "Maschine" als Gefolgschaft zu haben.7 Die Übertragbarkeit der Begriffe wird im Wissenschaftsdiskurs analytisch beleuchtet, im Me-

\footnotetext{
7 "Aber es gibt nur die Wahl: Führerdemokratie mit 'Maschine' oder führerlose Demokratie, das heißt: die Herrschaft der 'Berufspolitiker' ohne Beruf, ohne die inneren, charismatischen Qualitäten, die eben zum Führer machen" (Weber 1919: 47-48).
} 
diendiskurs jedoch wenig differenziert zur Argumentation für eine heutige Notwendigkeit von Charisma in der Politik herangezogen.

(3) Postulierend: Intertextuelle Bezüge zu Max Weber werden im Diskurs jedoch nicht immer gekennzeichnet. Im nächsten Beispiel argumentiert ein Journalist, der sich sozusagen auf das Thema Charisma spezialisiert hat, vor einem ebenfalls normativen Redehintergrund pro Charisma: Das Hauptargument liegt in der "guten Politik", die Charismatiker in Demokratien ausüben:

Das Geheimnis politischen Handelns besteht für den Soziologen Niklas Luhmann darin, dass mit jeder Entscheidung eine neue Geschichte beginnt. Gute Politik lebt also weniger vom peniblen Durchrechnen aller Möglichkeiten als von situativer Intelligenz. Charisma und Instinkt gehören ebenfalls zum Anforderungsprofil. In dieser Hinsicht sind Politiker Spitzenfußballern durchaus ähnlich. (...) Dieses ureigene Vermögen der Politik aus Augenmaß und Kompetenz ist zuletzt unzureichend zur Geltung gekommen.

(Nutt 2011)

Neben Weber ("Augenmaß") zieht der Akteur mit Niklas Luhmann eine weitere Autorität zur Unterstützung seiner These heran, die dem "Geheimnis" in der Politik auf die Spur kommen will. Die Gesamtargumentation lehnt sich jedoch an Webers Polarisierung an: In dem Artikel zieht der Autor zunächst "die neuen Regierungen in Griechenland und Italien", die er als "die neue Macht der Technokraten" bezeichnet, in Zweifel:

Montis Regierung der Fachleute steht nicht zuletzt für das hohe Risiko einer partiellen Selbstaussetzung der Demokratie.

(Ebd.)

Die italienische Regierung wird als schädlich für die Demokratie konstruiert, da sie aus Experten und eben nicht aus Charismatikern besteht. Analog zu Weber wird hier ein SchwarzWeiß-Muster aus "schlechten, weil rationalen Experten" (penibles Durchrechnen aller Möglichkeiten) einerseits und "guten Charismatikern" andererseits postuliert, sodass Charisma in diesem Vergleich als Garant für die lebendigere (vitalisierende) und damit bessere Politik in der Demokratie überzeugen kann. Auch hier liegt der Argumentation neben der vereinfachenden Polarisierung ein generalisierendes Kausalschema zugrunde, da Charisma als materielle Ursache für gute Politik angesehen wird.

Nicht erklärt wird, was unter Charisma zu verstehen ist. Der Ausdruck wird als Schlüsselwort neben Instinkt, situative Intelligenz, Augenma $\beta$ und Kompetenz gestellt, wenngleich Charis$m a$, eben weil es nicht konkret vorstellbar ist, das tragende Element in dieser Aufzählung notwendiger Eigenschaften bildet und diese dadurch aufwertet. Darüber hinaus verharmlost der Vergleich des Politikers mit dem Fußballer die charismatische Macht, als wäre Politik lediglich ein Spiel und die Bürger Zuschauer. Mystifizierung auf der Ebene der Modalität, da nur über die Kollokation erahnbar ist, was mit Charisma gemeint ist, und Veralltäglichung der Eigenschaft auf der inhaltlichen Ebene, stützen die These, dass Charismatiker für demokratische Prozesse besser geeignet sind als Technokraten. Die Konklusion auch hier: Charisma ist notwendig für demokratische Prozesse.

Dass es sich bei Charisma in der Politik nicht um ein harmloses Fußballspiel handelt, sondern um ernste Aushandlungsprozesse von Identität, Prestige und Stigma, zeigen die folgenden Beispielaussagen. 
(4) Postulierend: Im Vorfeld der Bundespräsidentenwahl 2010 war Joachim Gauck mehrheitlich als Charismatiker konstruiert worden, so z. B. in der Online-Ausgabe der Süddeutschen Zeitung (16.06.2010). "In Christian Wulff und Joachim Gauck stehen sich der Amtspolitiker ohne Charisma und der Nichtpolitiker mit Charisma gegenüber". Die Polarisierung macht aus einem normalen Politiker im Vergleich mit einem Charismatiker automatisch einen negativ behafteten Amtspolitiker. Nach der Bundespräsidentenwahl, die Wulff für sich entschied, gab es Stimmen, die das Scheitern Gaucks bedauerten:

Gleichwohl wäre die Wahl Gaucks allein deshalb schon wünschenswert gewesen, weil dann die Sehnsüchte nach einem charismatischen Politiker demokratisch gebunden gewesen wären. So aber hinterlässt das Fehlen charismatisch handelnder Politiker ein Vakuum, und eine Anfälligkeit der Deutschen für populistische Vereinfacher besteht fort.

(Steg 2010)

Das Fehlen von Charisma wird in einem Kausalschema als materielle Ursache für das Fortbestehen einer Anfälligkeit für Populisten konstruiert. Es wird polarisiert zwischen einem guten Charisma als Heilmittel gegen einen schlechten Populismus. Gleichwohl reagieren "die Deutschen" beides Mal emotional auf einen Politiker: indem sie Sehnsucht nach einem Charismatiker haben und indem sie anfällig sind für die vereinfachenden Reden des Populisten, die sie rational nicht durchschauen können. Demokratie und Charisma sind in dieser Logik notwendig aufeinander angewiesen: Die Demokratie benötigt Charismatiker, damit das Volk nicht auf nicht-legitimierte Politiker mit starker Rhetorik hereinfällt. Diese Logik präsupponiert jedoch, dass der gewählte charismatische Politiker eindeutig als "gut" identifizierbar ist und es aufgrund seiner demokratischen Gebundenheit auch bleibt. Fraglich ist zudem, ob die gleichen Wähler, die anfällig sind für Populisten, dann bei Wahlen in der Lage sind, gute Charismatiker von schlechten Populisten zu unterscheiden. Bei der Wahl des Bundespräsidenten liegt die Verantwortung hierfür bei den Politikern. Thomas Stegs Aussage, die Gauck zum Retter vor den Populisten stilisiert, ist daher auch eine Selbstaufwertung. Als Politiker, der in der Lage ist, den Unterschied zu erkennen, stellt er sich über das anfällige Gros der Deutschen, das er damit abwertet.

Dass der Unterschied zwischen einem guten Charismatiker und einem schlechten Populisten im Diskurs nicht eindeutig gezogen wird, zeigen Diskursaussagen über den verstorbenen österreichischen Politiker und Landeshauptmann von Kärnten, Jörg Haider, der in den Medien häufig als Populist, hier aber als Mann mit Charisma bezeichnet wird:

Jörg Haider ist tot. Wer ihn kannte, konnte von seinem Charme und seinem Charisma eingenommen sein. Und diese Gabe brachte ihm den politischen Erfolg, sie machte ihn so gefährlich.

(Stegemann 2008)

Haiders charismatische "Gabe" war demokratisch gebunden, gerade deshalb wird sie in dieser Diskursaussage als "gefährlich" eingestuft. Aussagen hingegen, die Charisma als notwendig für demokratische Prozesse erachten, implizieren, dass diese vermeintliche Gabe ausnahmslos positive Wirkungen hat. Das Haider-Beispiel macht deutlich, dass dies eine vereinfachende Perspektive ist, dass Charisma und Populismus Diskursphänomene sind, die nicht getrennt voneinander untersucht werden können, da sich ihre diskursiven Bedeutungen überlagern, es zu semantischen Verschmelzungen kommt: So schrieb FAZ am 25.12.2014 über den AfDGründer Bernd Lucke im Teaser eines Artikels: "Bernd Lucke ist das Gegenteil eines charis- 
matischen Populisten." Populisten können demnach gleichzeitig Charismatiker sein und Charismatiker Populisten. Wenn Bernd Lucke nun das Gegenteil ist, ist er dann weder charismatisch noch populistisch? Der Satz "Bernd Lucke ist kein charismatischer Populist" hätte womöglich mehr Klarheit gestiftet; denn genau darauf läuft die Argumentation in dem Text hinaus: Lucke ist nicht charismatisch, dennoch ist er ein erfolgreicher Populist. Implizit wird hier Charisma als Notwendigkeit für einen erfolgreichen Populismus verstanden, und Lucke bildet eine Ausnahme.

Ebenso wie Haider und Lucke vereint auch der (seinerzeit amtierende) Parteichef der Linkspartei, Oskar Lafontaine, beide Zuschreibungen auf sich. Spiegel online zitiert 2008 Aussagen von Helmut Schmidt aus der Bild am Sonntag, in denen Schmidt Lafontaine als Charismatiker und als Populisten bezeichnet:

Wir sehen jetzt in Amerika, wie ein junger Mann, Barack Obama, allein mit Charisma zu einer nationalen Figur wird. Dabei darf man nicht vergessen, dass Charisma für sich genommen noch keinen guten Politiker ausmacht", so Schmidt und begeht sodann einen Tabubruch: Auch "Adolf Nazi" sei ein charismatischer Redner gewesen. "Oskar Lafontaine ist es auch". Später in dem Interview vergleicht er Lafontaine auch noch mit dem französischen Rechtspopulisten Jean-Marie Le Pen. "Der eine ist links, der andere ist rechts. Aber vergleichbare Populisten sind Lafontaine und Le Pen schon", sagte Schmidt der "BamS.

(Römmele 2008)

Nach dem Interview in der BamS konstruierten die Medien Schmidts "Tabubruch" als solchen. Sie stellten, wie auch hier Spiegel online, das "normative Vergleichsschema" (Kienpointner 1993: 286) heraus, das dann folgender Logik folgt: Wenn sich Hitler und Lafontaine in dem Kriterium des charismatischen Redetalents ähnlich sind, so sind sie "im Normalfall diesbezüglich gleich/ähnlich zu bewerten bzw. zu behandeln" (Kienpointner 1993: 286). Der Vergleich beider Politiker wird in der Aussage Schmidts nicht direkt mittels vergleichender Konjunktionen gezogen, sondern indirekt über das Konjunktionaladverb auch. Dieser Vergleich zwischen Hitler und Lafontaine (eigentlich zwischen Obama einerseits und Hitler und Lafontaine andererseits), in dem es nicht mehr um die Verbindung von Charisma und Eloquenz geht, sondern um Unberechenbarkeit, Gefährlichkeit, Fanatismus usw., wurde von den Medien durch verkürzte Zitate selbst gehypt und dann negativ evaluiert. Nazi-Vergleiche werden in den deutschen Medien weitgehend als unangemessen betrachtet, und sie diffamieren eher den Autor als das herangezogene Vergleichsobjekt.

In der Süddeutschen Zeitung wurde später Bezug nehmend darauf Roman Herzog zitiert, der Lafontaine wieder ein positives Charisma zuspricht, ihn sozusagen rehabilitiert:

Der ehemalige Bundespräsident Roman Herzog (CDU) hält Oskar Lafontaine, den Parteichef der Linkspartei, für den einzigen charismatischen Politiker in Deutschland - und meint es offenbar als Kompliment. [...] Im September hatte der Altbundeskanzler Helmut Schmidt (SPD) seinen ehemaligen Parteikollegen Lafontaine wegen seines Redetalents indirekt in eine Reihe mit Adolf Hitler gestellt.

(Römmele 2010)

Altbundeskanzler Schmidt selbst wird in den Medien als Alterscharismatiker bezeichnet. Doch seine Reden werden zunehmend kritisch betrachtet, sein Alters-Charisma als Freibrief für Entgleisungen kritisiert: "Helmut Schmidt ist Alterscharismatiker. Deshalb stößt er mit jeder noch so unsinnigen Äußerung auf Gehör." (Süddeutsche, 14.04.2014). Wenn Roman 
Herzog Lafontaine (entgegen der von den Medien hervorgehobenen Aussage Schmidts) ein positiv besetztes Charisma zuspricht, so ist dies Ausdruck eines medial inszenierten Charisma-Deutungskampfes zweier ebenfalls durch die Medien konstruierter CharismaTräger. Grundsätzlich stellen sich folgende Fragen: Wem nützen oder schaden (De)Charismatisierungen bzw. positive/negative Evaluationen von Charisma? Welche sprachlichen Mittel sind erlaubt? Zu welchem Zweck werden in den Medien CharismaVergleiche inszeniert?

Werden Wählbarkeit und Wirksamkeit (nur) dem charismatischen Politiker zugeschrieben, so wird deutlich, dass Charisma eine Eigenschaft oder Wirkung ist, die für demokratische Prozesse als unerlässlich betrachtet wird. Während bei Weber und Freud das Charisma theoretisch aus Krisen hervorgeht und erst Ordnung schafft, so liegt hier mit Richard Sennett (1983: 312) die Annahme zugrunde, dass Charisma die Ordnung selbst ist, jedoch ohne die von Sennett konstatierten negativen Implikationen. Je nachdem, wie erfolgreich diese Glaubenskonstruktion ist, beeinflusst der Charisma-Glaube real-existierende Machtverhältnisse, stabilisiert sie, verändert sie. Wenn beispielsweise Lafontaine das zuvor konstruierte Charisma erfolgreich abgesprochen oder sein positives Charisma in ein negatives verwandelt wird, so kann diese Identitätskonstruktion der Partei bei ihrem Versuch schaden, vom ausgegrenzten linken Rand in die Parteienmitte zu wandern.

Im Diskurs finden sich nun auch Aussagen, die Charisma relativierend, abhängig von den gesellschaftlichen Umständen, positiv bewerten. Der Redehintergrund ist hier als "circumstantiell" zu benennen, da hier "nicht von dem gesamten Wissen, sondern speziellen Umständen und Gegebenheiten ausgegangen wird" (Zifonun 1997: 1883):

2) Charisma ist eine für demokratische Prozesse nützliche Eigenschaft von Politikern.

(5) Die Logik der Aussagen dieser Kategorie kann salopp mit "Charisma ist nicht notwendig, aber es hilft" paraphrasiert werden. Das folgende Beispiel verdeutlicht diesen Zusammenhang.

Guttenbergs Abgang hat eine Lücke gerissen. Denn die Sehnsucht nach Charismatikern nimmt zu. [...] Wäre die Identifikation mit Volkstribunen nicht besser geeignet, das plebiszitäre Element und die Attraktivität der Politik zu stärken? [...] Neu ist jedoch, dass Hoffnungen auf eine Verbesserung der Demokratiequalität und -legitimität derart stark auf einzelne Personen abstellen, sei es Obama oder jetzt zu Guttenberg.

(Linden 2011)

Nachdem Karl Theodor zu Guttenberg des Plagiats überführt wurde und er als Verteidigungsminister zurücktrat, arbeiteten die Medien die vorhergehende Charismatisierung Guttenbergs auf. Aus Sicht der taz ist es die "Sehnsucht nach Charismatikern", die ursächlich ist für Guttenbergs Charismatisierung und die eine übertriebene Identifikation mit einzelnen Politikerpersönlichkeiten befördert. Gleichzeitig wird als Grund für die Identifikation der Wunsch nach einer "Verbesserung der Demokratiequalität und -legitimität" angegeben. Die taz kritisiert daher nicht die Sehnsucht nach Charisma, sondern fragt im Konjunktiv nach einer besseren Nutzbarmachung dieser Sehnsucht. Die Beteiligung des Volkes an der Demokratie soll gestärkt werden, Politik soll attraktiver werden. Dahinter versteckt sich der Wunsch, nicht kontrollierbare emotionale Prozesse in rationale Kanäle zu leiten, die der Demokratie nützen, nicht schaden. 
Der Wunsch nach einem nützlichen Charisma verbindet sich hier mit der Auffassung von einer Demokratie, die in ihrer jetzigen Form überwunden werden muss. Diese Form ist die Postdemokratie (vgl. Crouch 2008). Das postdemokratische, postmoderne Charisma wird somit zu einem möglichen Heilmittel gegen die Politikverdrossenheit der Bürger und gegen einen oberflächlichen Personenkult. Die Macht der privilegierten Elite soll sich in dieser weltverbessernden Narration nicht gegen das Volk richten, sondern wieder die Macht des Volkes repräsentieren. Im Gegensatz zur ersten Aussagen-Kategorie, in der Charisma als normales demokratisches Element betrachtet wird, wird Charisma in den Aussagen der zweiten Kategorie als systemtranszendierendes Element betrachtet, werden demokratische Prozesse in einer differenzierteren Weise reflektiert.

Zudem gibt es Diskursaussagen, in denen Charisma aus verschiedenen Gründen für unvereinbar mit demokratischen Prozessen gehalten werden:

3) Charisma ist eine für demokratische Prozesse unpassende oder gefährliche Eigenschaft von Politikern, daher sind Charisma und Demokratie unvereinbar.

Diese Kategorie lässt sich in drei Varianten aufteilen: 1. Vor einem normativen Redehintergrund wird Charisma generalisierend als unvereinbar mit demokratischen Prozessen betrachtet. 2. Es wird zunächst die Abwesenheit von Charisma in der deutschen Politik konstatiert und bedauert, dann aber relativierend für positiv erachtet. Der Redehintergrund ist circumstantiell, da zumeist situative Gründe wie z. B. Krisen als Argument gegen Charisma angegeben werden. 3. Die Abwesenheit von Charisma in der deutschen Politiklandschaft wird als positiv gewertet, da es sich hierbei nur um die "boulevardeske Säkularisierung der göttlichen Begabung zum Glamour" (taz, 10.03.2011) handelt. Aufgrund von negativen Erfahrungen mit Charisma wird hier ebenfalls vor einem circumstantiellen Redehintergrund gewertet.

(6) Normativ: Normative, Charisma negativ bewertende Konstruktionen sind in dem ausgewerteten Teilkorpus sehr selten. Es wurden nur wenige Aussagen gefunden, die Charisma rundweg ablehnen.

Aber auch Charisma beruht auf einer Illusion, wie der Glaube an eine heile Welt. Niemand ist perfekt. Beim charismatischen Politiker hat es nur den Anschein. Er trügt. [...] Ich habe nicht das Bedürfnis, von Heiligen regiert zu werden.

(Martenstein 2011)

Auch diese Aussage entstand im Zuge der medial verarbeiteten Enttäuschung über den einstigen Hoffnungsträger Guttenberg. Die Absurdität der Charismakonstruktion als "Illusion" wird mit einem Vergleichsschema gestützt. Während Diskursakteure mehrheitlich bemüht sind, ihre Charismakonstruktionen mittels sakralisierender, mystifizierender oder rationalisierender sprachlicher Mittel zu legitimieren, wird hier entsakralisiert ("Glaube", "Heilige") und entmystifiziert ("Illusion", "Anschein"). Es ist der Versuch einer Re-Rationalisierung des Charisma-Diskurses, bei der die Ich-Perspektive des Akteurs der Argumentation zusätzliches Gewicht verleiht. Die Negation eines "Bedürfnis[ses], von Heiligen regiert zu werden", wertet den Akteur ebenso auf, wie im umgekehrten Fall die Fähigkeit, einen Charismatiker erkennen zu können. Während letztere über die Charismatisierung eine sprachliche Verbindung zum Charismatiker selbst schafft und man sich damit vom Rest der für Populisten anfälligen Deutschen abgrenzt, geschieht hier die Selbstaufwertung über die generalisierte Abwertung von Charisma. Das rationale Erkennen des Charismas als "Illusion" steht gegen eine emotionale 
Hingabe, die nicht nur die anfälligen Deutschen, sondern auch charismakonstruierende Politiker und Medien kritisiert.

"Ich halte das für ein vordemokratisches Denken, dass das politische Führungspersonal charismatisch qualifiziert sein müsste", sagt der Marburger Soziologe Dirk Kaesler.

(Storost 2010)

Auch diese Aussage des Soziologen Dirk Kaesler kann zu einem Charisma-Gegendiskurs gezählt werden. Es ist dies wie bei Römmele (im Folgenden) eine Expertenargumentation, die Charisma nach einem normativen Definitionsschema (vgl. Kienpointner 1993: 251) implizit bewertet: Wenn die "charismatisch[e]" Qualifizierung von Politikern als "vordemokratisches Denken" definiert ist, dann ist die negative Bewertung von Charisma gerechtfertigt.

Es verbirgt sich hinter diesen Diskursaussagen eine andere Bedeutung von Charisma, als sie bei den Aussagen der ersten und zweiten Kategorie angenommen werden kann. Charisma bedeutet hier nicht harmlos "Augenmaß" oder "Ausstrahlung", auch nicht mystifiziert "Aura". Die semantische Relation von Charisma und Illusion als ursächlicher Verbindung macht den diskursimmanenten Glaubenssprung luzid. Charisma gehört nach dieser Definition der Subsinnwelt der persönlichen, subjektiven Transzendenzerfahrung an, die nicht in die Politik gehört. Wenn Charisma mit vordemokratisch attribuiert wird, so muss, um dies zu verstehen, Webers Herrschaftstypologie als inhärentes Wissen herangezogen werden. Herrschaftsanspruch und Irrationalität stehen in Verbindung mit einem vordemokratischen, vormodernen Herrschaftscharisma, das in Bezug auf die parlamentarische Demokratie systemfremd bis systemgefährdend ist. Charisma ist auch hier die Ordnung, allerdings nicht als Teil eines demokratischen Systems, sondern sie ist

außeralltägliche Hingabe an die Heiligkeit oder Heldenkraft oder die Vorbildlichkeit einer Person und der durch sie offenbarten und geschaffenen Ordnungen.

(Weber 1922: 124, Hervorhebung P. S.)

Bei dieser traditionellen Charisma-Auffassung ist das Volk nicht Souverän; Charisma und Demokratie sind unvereinbar.

(7) Circumstantiell: Konstatiert wird zunächst die Abwesenheit von Charisma, indem erneut der Allgemeinheit ein unerfüllter Wunsch nach charismatischer Führung unterstellt wird. Daraufhin erfolgt ein Argumentieren contra Charisma, das als Ausdruck einer Resignation interpretiert werden kann.

Die Reaktionen auf Barack Obamas Besuch in Europa haben wieder einmal unseren Wunsch nach charismatischer Führung gezeigt. Unser politisches System macht es aber schwer, uns diesen Wunsch selbst zu erfüllen - und vielleicht ist das auch gut so. [...] In parlamentarischen Demokratien geht zum Glück kein Weg an den Parteien vorbei. Sie sind die zentralen Ausbildungsstätten der Politiker, das "training on and for the job" findet hier statt. In Zeiten, in denen Politik immer vielschichtiger und komplexer wird, braucht es diese solide Basis. Hier werden weniger charismatische Führungsqualitäten gefragt, sondern Sachkenntnis, einen hohe Problemlösungskompetenz und Schnittstellenmanagement - also im Sinne Max Webers "rationale" Qualitäten.

(Römmele 2009)

Römmele argumentiert diesmal mit den Anforderungen des demokratischen Systems gegen Charisma. Sie verwendet hier den Komplexitäts-Topos gegen den Charismatiker. Vor dem 
Hintergrund einer "vielschichtiger[en] und komplexer[en]" Politik als circumstantielle Ausgangsdaten steht Charisma einer politischen Karriere und einem Nutzen für das System im Weg. "Charismatische Führungsqualitäten" werden antonym konstruiert zur "solide[n] Basis", die stattdessen benötigt wird. Die bewirkenden Ursachen für den Erhalt der Basis sind in diesem Schema "Sachkenntnis", "Problemlösungskompetenz" und "Schnittstellenmanagement". Die Akteurin argumentiert zudem mit Max Weber im Autoritätsschema contra Charisma (ein Jahr später, s. o., präsupponiert ihre Aussage zum Spezialfall des Bundespräsidenten, dass zumindest dieser aber Charisma benötigt). Das ist insofern bezeichnend, als sie sich nicht wie Kaesler auf den vordemokratischen Herrschaftsanspruch beruft, sondern auf die besseren "rationale[n]" Fähigkeiten des uncharismatischen Berufspolitikers. Mit Sennett (1983: 312) könnte leicht dagegen vorgebracht werden, dass Römmele, "Weber und Freud die charismatische Persönlichkeit mit Leidenschaftlichkeit und Illusion gleichsetzen und ihr die Rationalität gegenüberstellen", sie damit aber eine "Verkennung der Rationalität selbst" bekunden. "Sie sitzen der Illusion auf, Rationalität sei der Stiftung von Unordnung konträr".

Rationalität, so argumentiert Römmele, ist ein Heilmittel gegen die Komplexität der Politik. Roman Herzog (s. o.) führt den Komplexitäts-Topos im Gegenteil als ein Argument für den charismatischen Politiker an: Für eine tiefgründige Analyse ist keine Zeit, deshalb ist der im Augenblick handelnde Politiker gefragt, der aber ebenso wenig irrational handelt. Allerdings bildet Rationalität bei Römmele die zweite Wahl: Demokratisches System und Komplexität lassen keinen Charismatiker zu, dieser wäre unter anderen (amerikanischen) Bedingungen wünschenswert. Die Abwesenheit von Charisma ist hier systemkonservierend; Charisma und Demokratie sind zwar nicht unvereinbar, aber unpassend.

(8) Circumstantiell: Das Fehlen von Charisma in der deutschen Politik wird unter bestimmten systemimmanenten Voraussetzungen als besser erachtet.

In Zeiten der Mediendemokratie versteckt sich gerade hier die Gefahr des Charismatischen in der Politik. Wo Charisma und Leistung nicht korrespondieren, bleiben schnell Desillusionierung und politische Apathie zurück.

(Burmester 2014)

Charisma wird in diesem thematisierenden Text als "Gefahr" betrachtet, wenn es nicht mit "Leistung" einhergeht. Somit wird Charisma unter diesen Bedingungen in einem Kausalschema zur materiellen Ursache für typische Probleme der Postdemokratie: "Desillusionierung und politische Apathie". Die besondere Form der Mediendemokratie wird hier mitverantwortlich gemacht für die Gefahr, zu große Hoffnungen an eine Person und ihre Fähigkeiten zu knüpfen, die dann nicht erfüllt werden. Mediendemokratie als eine Form der "Medialisierung der Politik" (Tänzler 2007: 122) beschreibt somit eine Art Nährboden für substanzlose Charismatiker. Charisma ist aus dieser Perspektive nur mediale Inszenierung und damit Ausdruck von Medienmacht. Dieser Haltung liegt grundsätzlich die Annahme zugrunde, es habe früher ein wertgebundenes Charisma gegeben, das jedoch einem postmodernen "PseudoCharisma" (Lenze 2002) gewichen ist. Diskursaussagen dieser Art konstruieren analog zu Sennett (1983: 306) eine Art "narkotisches Charisma", das "Apathie" schürt. Aus Sicht der Mediendemokratie ist dieses Pseudo-Charisma systemimmanent, für die regulären demokratischen Prozesse, die kaum davon zu separieren sind, systemgefährdend. 


\section{Konzeptionelle Ausdifferenzierung der Begriffe Charisma und Demokratie}

Folgende tabellarische Übersicht verdeutlicht noch einmal den Zusammenhang von Charisma und Demokratie im Diskurs, wie er aus den acht untersuchten Beispielen hervorgeht.

\begin{tabular}{|c|c|c|c|}
\hline DHARISMA & $\begin{array}{c}\text { PARLAMENTARISCHE } \\
\text { DEMOKRATIE }\end{array}$ & POSTDEMOKRATIE & $\begin{array}{c}\text { MEDIEN- } \\
\text { DEMOKRATIE }\end{array}$ \\
\hline $\begin{array}{c}\text { DEMOKRATISCHES CHA- } \\
\text { RISMA }\end{array}$ & $\begin{array}{c}\text { systemimmanent } \\
\text { ex negativo: systemkon- } \\
\text { servierend }\end{array}$ & & \\
\hline $\begin{array}{c}\text { POSTDEMOKRATISCHES } \\
\text { CHARISMA }\end{array}$ & systemfremd & system- \\
transzendierend & & \\
\hline HERRSCHAFTSCHARISMA & systemgefährdend & & systemimmanent \\
\hline PSEUDO-CHARISMA & & & \\
\hline
\end{tabular}

Tabelle 1: Quelle: Eigene Darstellung

Als Demokratisches Charisma wird hier dasjenige Charisma bezeichnet, das Akteure wie selbstverständlich und unreflektiert konstruieren und das die normalen Funktionen einer parlamentarischen Demokratie am Laufen hält. Es ist daher eine Art normales Charisma, das sich in das System einfügt, sogar notwendig für gute Politik ist. Ex negativo, das heißt, bei seiner Abwesenheit, wird eben dieses normale demokratische Charisma aber auch als systemkonservierend verstanden. Wenn es fehlt, ruft es automatisch Berufspolitiker mit langjähriger Erfahrung auf den Plan, die in bestimmten Zeiten besser geeignet sind, die politischen Geschicke zu lenken.

Wenn Diskursakteure Charisma nicht als normales und notwendiges demokratisches Element betrachten, sondern als ein Mittel dafür, die Bürger wieder für Politik zu begeistern, müde Demokratien munter zu machen, wie es im Titel dieses Beitrags heißt, so kann dieses Charisma als ein postdemokratisches Charisma aufgefasst werden. Dieses Charisma ist ein Heilmittel in Zeiten der Postdemokratie, soll dieses politische System also transzendieren. Der Unterschied zum normalen demokratischen Charisma liegt demnach in seiner Funktion und in seiner Relation zum Demokratiebegriff, der ein anderer, erweiterter ist.

Wird Charisma als unvereinbar mit der Demokratie betrachtet, weil es vordemokratisch ist, so impliziert dieser Charismabegriff den von Weber stammendenden Herrschaftstypus. Charisma ist dann ordnungsstiftend, wenn sich das Volk einem Führer unterordnet. Da dieses Konzept der Idee von einer Volkssouveränität widerspricht, ist das Herrschaftscharisma systemgefährdend, weil es vormoderne Zeiten einläutet. Es bleibt dann aber zu fragen, wie diese Auffassung von Charisma mit der den Diskurs dominierenden Auffassung von einem demokratischen Charisma vereinbar ist. Diskursakteure, die Charisma als systemgefährdend betrachten, sehen entweder das vordemokratische Herrschaftspotenzial in charismatisierten Politikern, die sich gewissermaßen über demokratische Strukturen hinausheben. Oder sie tun das 
normale Charisma als reine Form der Ausstrahlung ab, das mit einer charismatischen Machtbeziehung im Sinne Webers wenig gemein hat.

\section{$5 \quad$ Zusammenfassung}

Die Ausführungen haben gezeigt: Sprachlich-mediale Charismakonstruktion kann nicht ohne ihre Verbindung zur diskursiven Konstruktion von Demokratie gedacht werden. Die sprachliche Konstruktion von Charisma ist dabei in vielerlei Hinsicht heterogen. Grundsätzlich zeigt sich Charisma als ein Ausdruck, dessen Bedeutung schwer vertextbar ist und es auch sein soll. Sakralisierende (z. B. religiöse Metaphern) und mystifizierende sprachliche Mittel (z. B. Reihung vager Hochwertwörter) wirken einer Demokratisierung der charismatischen Befähigung (vgl. Schneider 2008) entgegen. Charisma ist zwar überall im Diskurs präsent, aber es soll nicht jedem gehören.

Was Diskursakteure mit dem Ausdruck Charisma meinen, hängt von ihrer jeweiligen Argumentation ab. Hat ein Politiker keine Chance, die anstehende Wahl zu gewinnen, so liegt dies daran, dass er kein Charisma hat. In Zeiten, die durch Komplexität geprägt sind, ist der charismatische Politiker die Lösung, weil er spontan handeln kann, oder er ist ein Problem, weil er unüberlegt handelt, wo rationale Qualitäten gefragt sind. Heute ist ein Politiker ein charismatischer Redner, morgen ist er der Grund für Apathie und Desillusionierung. Ob ein eloquenter Redner ein guter oder böser (weil populistischer) Charismatiker ist, ob er überhaupt charismatisch oder nicht vielmehr populistisch agiert, hängt im Deutungskampf von der Wertung des schreibenden Diskursakteurs ab. Diese Wertungen können jedoch auf ihn zurückfallen und gegebenenfalls zu einem Diskursereignis werden, das De-Charismatisierungen der eigenen Person nach sich zieht. Charismakonstruktion ist ein sprachliches Machtspiel.

Auch bezüglich des ontologischen Status sind sich die Diskursakteure uneinig. Ist Charisma ein Faktum oder eine Illusion? Jedoch sind Stimmen, die die Existenz von Charisma anzweifeln, selten. Relative Einigkeit besteht textübergreifend bezüglich der Motivation der Charisma-Gläubigen, die häufig generalisierend konstruiert werden als die Deutschen. Der Sehnsuchts-Topos (Sehnsucht, Wunsch, Bedürfnis) ist hier sowohl ein Argument pro als auch contra Charisma. Mal ist der Wunsch nach Charisma verständlich, mal ist er gefährlich, mal ein Irrglaube. Die Schriften Max Webers werden zur rationalisierenden Erklärung herangezogen, um der jeweiligen Argumentation eine wissenschaftliche Pseudo-Tiefe zu verleihen. Dabei wird polarisiert zwischen einem Berufspolitiker und einem charismatischen Führer. Ein leidenschaftlicher, erfolgreicher Politiker ohne Charisma scheint kaum denkbar in dieser Schwarz-Weiß-Konstruktion. Der Politiker ist entweder erfolgreich, dann hat er Charisma wie Kubicki oder Lindner, oder er ist es nicht, dann heißt er Rösler. Thematisierende und Okkurrenz-Texte haben je nach Diskursereignis, auf das sich die Charisma-Konstruktion bezieht (z. B. Wahlen), Konjunktur; metasprachlich-reflexive Texte, die Charisma als Ergebnis einer Zuschreibung verstehen, sind selten.

Die Interpretation der ausgewählten Diskursaussagen hinsichtlich des Charisma-DemokratieZusammenhangs hat drei grundlegende Einstellungen deutlich gemacht. So wird Charisma als notwendig oder abgeschwächt als nützlich für die Demokratie betrachtet, je nachdem ob ein normativer oder circumstantieller Redehintergrund vorliegt. Ebenfalls vor einem normativen Redehintergrund wird eine Unvereinbarkeit von Charisma und Demokratie konstatiert, die 
vor einem circumstantiellen Hintergrund auch abgeschwächt als "unpassend" aufgefasst wird. Diese verschiedenen Einstellungen offenbaren jeweils eine andere Auffassung von Charisma, aber auch von Demokratie. Somit zeigt die Re-Konstruktion von politischem Charisma im Diskurs vor allem, dass die in der Soziologie gebräuchlichen Charisma-Auffassungen (z. B. Herrschaftscharisma, Pseudo-Charisma) im Mediendiskurs parallel existieren. Die Diskursaussagen zeigen, wie eine Gesellschaft den Begriff tatsächlich verwendet und demnach, was Charisma für sie bedeutet. Die Heterogenität in der Ausdrucksverwendung ist dabei ein wichtiger Indikator dafür, dass das semantische Feld in Bewegung ist. Dies wiederum zeigt an, dass der Charisma-Begriff ein wichtiges Instrument im politischen Diskurs ist, um soziale Identitäten und mit diesen soziale Hierarchien zu konstituieren, die auf Deutungsmacht beruhen. Ein als charismatisch konstruierter Politiker ist nicht einfach nur ein guter oder eloquenter Politiker, sondern an dieser Konstruktion hängen elementare demokratische Strukturmerkmale: die Befindlichkeit der Gesellschaft, die Einstellung gegenüber dem Politiksystem, gegenüber einer Partei, gegenüber einem bestimmten Politiker oder der Politikerkaste, und nicht zuletzt die Konstruktion der Welt, in die der Charismatiker gesetzt wird, sei sie nun komplex und unübersichtlich, krisengeschüttelt oder medial verflacht. Charisma ist nicht nur eine sprachlich konstruierte Machtdisposition, sondern der Akt der sprachlichen Konstruktion selbst birgt im politischen Diskurs ein starkes Macht-Potenzial.

\section{Literatur}

Berger, Peter L./Luckmann, Thomas (2009): Die gesellschaftliche Konstruktion der Wirklichkeit. 22. Auflage. Frankfurt am Main: Fischer.

Bizeul, Yves (2009): Glaube und Politik. Wiesbaden: Verlag für Sozialwissenschaften.

Bornscheuer, Lothar (1976): Topik. Zur Struktur der gesellschaftlichen Einbildungskraft. Frankfurt am Main: Suhrkamp.

Breuer, Stefan (1991): Max Webers Herrschaftssoziologie. Frankfurt am Main: Campus.

Busse, Dietrich/Teubert, Wolfgang (Hrsg.): Linguistische Diskursanalyse: neue Perspektiven. Wiesbaden: Springer VS.

Crouch, Colin (2008): Postdemokratie. Frankfurt am Main: Suhrkamp.

Dudenredaktion (Hrsg.) (2015): Duden. Fremdwörterbuch. 11., vollständig überarbeitete und aktualisierte Auflage. Mannheim etc.: Dudenverlag.

Fasel, Christoph (2001): "Die Nase ist die Nachricht. Wie Menschen Medienereignisse machen". In: Häusermann, Jürg (Hrsg.): Inszeniertes Charisma. Medien und Persönlichkeit. Tübingen, Niemeyer: $55-66$.

Gebhardt, Winfried/Zingerle, Arnold/Ebertz, Michael N. (1993): "Vorwort". In: Gebhardt, Winfried/Zingerle, Arnold/Ebertz, Michael N. (Hrsg.): Charisma. Theorie - Religion - Politik. Berlin/New York, de Gruyter: V-VI.

Jentges, Erik (2010): Die soziale Magie politischer Repräsentation. Charisma und Anerkennung in der Zivilgesellschaft. Bielefeld: Transcript.

Kienpointner, Manfred (1992): Alltagslogik. Struktur und Funktion von Argumentationsmustern. Stuttgart/Bad Cannstatt: Friedrich Frommann.

Knoblauch, Hubert (2009): Populäre Religion. Auf dem Weg in eine spirituelle Gesellschaft. Frankfurt am Main/New York: Campus. 
Lenze, Malte (2002): Postmodernes Charisma. Marken und Stars statt Religion und Vernunft. Wiesbaden: Deutscher Universitäts-Verlag.

Luhmann, Niklas (2000): Die Religion der Gesellschaft. Frankfurt am Main: Suhrkamp.

Marschall, Christoph von (2011): "Charisma und politische Führung in den USA. Barack Obama - ein schwarzer Kennedy?". In: Bliesemann de Guevara, Berit/Reiber, Tatjana (Hrsg.): Charisma und Herrschaft. Führung und Verführung in der Politik. Frankfurt/New York, Campus: 53-76.

Otto, Rudolf (1917): Das Heilige: Über das Irrationale in der Idee des Göttlichen und sein Verhältnis zum Rationalen. Breslau: Trewendt/Granier.

Rappaport, Roy A. (1979): "The Obvious Aspects of Ritual". Auszüge und in deutscher Übersetzung. In: Belliger, Andréa/Krieger, David J. (Hrsg.) (2013): Ritualtheorien. Ein einführendes Handbuch. 5. Auflage. Wiesbaden, Springer VS: 189-208.

Schneider, Christoph (2008): "Charisma. Sinnproduktion durch Reflexionsanästhesie". In: Rychterová, Pavlína/Seit, Stefan/Veit, Raphaela (Hrsg.): Das Charisma. Funktionen und symbolische Repräsentationen. Berlin, Akademieverlag: 129-153.

Schütz, Alfred/Luckmann, Thomas (2003): Strukturen der Lebenswelt. Konstanz: UTB.

Sennett, Richard (1983): Verfall und Ende des öffentlichen Lebens. 2. Auflage. Frankfurt am Main: S. Fischer.

Spitzmüller, Jürgen/Warnke, Ingo (2011): Diskurslinguistik. Eine Einführung in Theorien und Methoden der transtextuellen Sprachanalyse. Berlin/Boston: de Gruyter.

Steyrer, Johannes (2011): "Jörg Haider - charismatischer Führer, narzisstische Persönlichkeit und Rechtspopulist". In: Bliesemann de Guevara, Berit/Reiber, Tatjana (Hrsg.): Charisma und Herrschaft. Führung und Verführung in der Politik. Frankfurt/New York, Campus: 77-101.

Tänzler, Dirk (2000): "Das ungewohnte Medium. Hitler und Roosevelt im Film". Sozialer Sinn 1: 93-120.

Tänzler, Dirk (2007): "Politisches Charisma in der entzauberten Welt". In: Gostmann, Peter/Merz-Benz, Peter-Ulrich (Hrsg.): Macht und Herrschaft. Zur Revision zweier soziologischer Grundbegriffe. Wiesbaden, Verlag für Sozialwissenschaften: 107-137.

Turner, Stephen (2007): "Charisma - neu bedacht". In: Gostmann, Peter/Merz-Benz, PeterUlrich (Hrsg.): Macht und Herrschaft. Zur Revision zweier soziologischer Grundbegriffe. Wiesbaden, Verlag für Sozialwissenschaften: 81-105.

Weber, Max (1919): Geistige Arbeit als Beruf. Zweiter Vortrag: Politik als Beruf. München/Leipzig: Duncker/Humblot.

Weber, Max (1922/1980): Wirtschaft und Gesellschaft. Grundriss der verstehenden Soziologie. 5., revidierte Auflage besorgt von Johannes Winckelmann, Studienausgabe. Tübingen: J. C. B. Mohr.

Wengeler, Martin (2013): "Historische Diskurssemantik als Analyse von Argumentationstopoi". In: Busse, Dietrich/Teubert, Wolfgang (Hrsg.): Linguistische Diskursanalyse: neue Perspektiven. Wiesbaden, Springer: 189-215.

Zifonun, Gisela/Hoffmann, Ludger/Strecker, Bruno (1997): Grammatik der deutschen Sprache. Berlin/New York: de Gruyter. 


\section{Korpusverzeichnis}

Burmester, Hanno (2014): "Charisma macht müde Demokratien munter". Berliner Republik. Das Debattenmagazin 3. www.b-republik.de/archiv/charisma-macht-muede-demokratienmunter [06.10.2014].

Linden, Markus (2011): "Germanys Next Topminister". TAZ. http://taz.de/DebattePopulismus/!5125400/ [02.07.2014].

Martenstein, Harald (2011): "Von Dummen will ich nicht regiert werden". Tagesspiegel. www.tagesspiegel.de/meinung/guttenberg-von-dummen-will-ich-nicht-regiertwerden/3862872.html [07.10.2014].

Nutt, Harry (2011): "Ein waghalsiges Experiment". Frankfurter Rundschau. www.fronline.de/politik/leitartikel-ein-waghalsiges-experiment,1472596,11173912.html [05.10.2014].

Römmele, Andrea (2009): "Warum haben wir keinen Obama?". Zeit online. http://blog.zeit.de/zweitstimme/2009/04/11/warum-haben-wir-keinen-obama/ [28.09.2014].

Römmele, Andrea (2010): "Deutschlands oberste Tigerente, oder: Zur (Aus-)Wahl des Bundespräsidenten". Zeit Online. http://blog.zeit.de/zweitstimme/2010/06/03/deutschlandsoberste-tigerente-oder-zur-aus-wahl-des-bundesprasidenten [06.10.2014].

Römmele, Andrea: (2008): "Altkanzler-Attacke: Schmidt vergleicht Lafontaine mit Hitler und Le Pen". Spiegel. www.spiegel.de/politik/deutschland/altkanzler-attacke-schmidtvergleicht-lafontaine-mit-hitler-und-le-pen-a-578116.html [21.10.2014].

Römmele, Andrea: (2010): "Der einzige Charismatiker". Süddeutsche Zeitung. www.sueddeu tsche.de/politik/herzog-ueber-lafontaine-der-einzige-charismatiker-1.428960 [24.12.2014].

Sigmund, Tomas (2012): "Lindner und Kubicki verbindet nur das Charisma". Handelsblatt. www.handelsblatt.com/meinung/kommentare/kommentar-lindner-und-kubicki-verbindetnur-das-charisma/6603834.html [04.09.2014].

Steg, Thomas (2010): "Die Bürger verspüren eine tiefe Sehnsucht nach Charisma". Handelsblatt. www.handelsblatt.com/meinung/kommentare/politikbetrieb-die-buerger-verspuereneine-tiefe-sehnsucht-nach-charisma/3483526.html [08.11.2014].

Stegemann, Hubert Kleine (2008): "Jörg Haider ist tot". Bonner General-Anzeiger. www.general-anzeiger-bonn.de/news/kommentare/Joerg-Haider-ist-tot-article181284.html [17.11.2014].

Storost, Ursula (2010): "Charisma in der Politik". Deutschlandfunk. www.deutschlandfunk.de/charisma-in-der-politik.1148.de.html?dram:article_id=180545 [11.09.2014]. 